\title{
Subsurface Imaging using Ground-Penetrating Radar Measurements
}

\author{
Nathan Goodman, Carl Leuschen, Richard Plumb and Chris Allen \\ University of Kansas, Radar Systems and Remote Sensing Laboratory \\ 2291 Irving Hill Road, Lawrence, KS 66045-2969 USA \\ TEL: 913/864-7735, FAX: 913/864-7789, E-mail: plumb@eecs.ukans.edu
}

\begin{abstract}
Much work has been done toward reconstructing the electrical parameters of an unknown buried object. Many methods, and accompanying results from simulations, have been presented. Most research in the area, however, has been in developing imaging algorithms that have been tested primarily through computer simulation. This paper presents a newly constructed facility for ground-penetrating radar experiments, as well as the proposed applications for such a facility. The test site consists of a sand-filled volume approximately 15 feet long, 13 feet wide, and 7 feet deep. The experimental setup consists of transmitters and receivers in several common configurations, such as the offset VRP, cross-borehole, and surface-to-surface configurations. The motivations for such an experimental site are presented, as well as the imaging algorithms intended for application. These algorithms include migration techniques, SAR processing, Born-iterative method, and diffraction tomography.
\end{abstract}

\section{INTRODUCTION}

Ground-penetrating radar has many applications ranging from detection of buried pipes to non-destructive probing for contaminants to mapping of the subsurface layers. Furthermore, the advantages of ground-penetrating radar over drilling and digging are tremendous. Ground-penetrating radar is less destructive and can provide more information than other methods of exploration. Ground-penetrating radar will also become less expensive and more powerful as technology and computing power progress. These reasons have sparked a growing interest in ground-penetrating radar algorithms that image the subsurface. However, very few of these algorithms have been applied outside the realm of computer simulation. It is this last fact that has motivated the groundpenetrating radar group at The University of Kansas in much of our recent work.

The lack of application of ground-penetrating radar in real field experiments has driven our current research toward developing techniques that can be successfully applied in the field. However, it is obvious that many difficulties would arise were the algorithms taken directly from the computer simulation environment to the field. For this reason a facility has been constructed that will allow for testing of many imaging approaches and algorithms in a controlled environment. Many common algorithms will be tested and compared. The results, computational power needed, ease of data acquisition, and other parameters will be investigated. The end result will be a thorough investigation of ground- penetrating radar subsurface imaging techniques, another step toward making ground-penetrating radar a more widely used method of subsurface exploration.

\section{EXPERIMENTAL FACILITY}

Work has just been completed on a fully automated test facility. A large concrete box was constructed with inner dimensions of 15 feet long by 13 feet wide by 7 feet deep. A platform provides easy access to the top of the box. The box is filled with sand providing a homogeneous background. Last, drains were included at the bottom of the box. These drains will be used in the future for experiments on imaging liquid contaminants and for providing an adjustable water table.

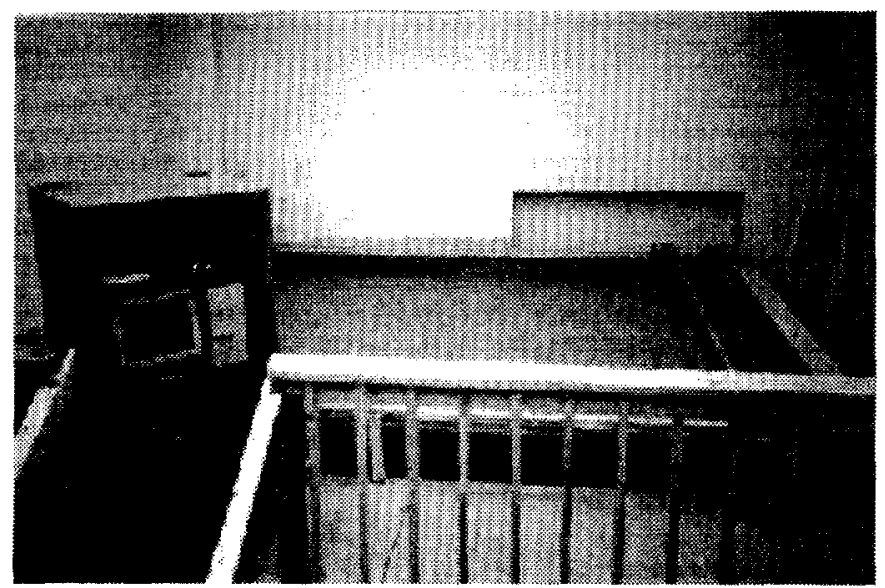

Experimental Facility and Test Equipment

Mounted on top of the box is a custom-designed gantry system. The system is constructed of extruded aluminum and spans the entire test area. Two independent gantries provide positioning for two antennas that can be placed at any two positions above the sand. Also, with another mechanical attachment, the antennas can be lowered into the sand, allowing for other ground-penetrating radar configurations that require the use of a borehole, such as the cross-borehole and offset VRP configurations. Last, one length of PVC pipe spans the entire box below the level of the sand. This opening allows a receiving antenna to be placed within the sand in order to verify our predictions of the fields radiated from more complicated antennas.

The gantry system is fully controllable with a personal computer. A 4-axis PC Servo-Motor Controller card was purchased and placed in a PC. The card drives four servo 
amplifiers independently, with each amplifier driving a different axis. Each gantry can be placed at any position above the sand with the exception that the two cannot cross each other. This limitation will be easily overcome through electronic switching of the antennas through software. Each axis is equipped with an optical encoder, and the Servo-Motor Controller uses this information to generate and execute any programmed motion profile with excellent accuracy. The entire experiment can be programmed and left alone.

\section{RADAR EQUIPMENT}

Experiments will be performed with two different radar systems. The first uses a Hewlett Packard 8753D Vector Network Analyzer. The network analyzer measures the desired reflection or transmission parameter. The network analyzer is fully controllable via a National Instruments GPIB Interface Board and Labview software. Commands and data can be read from and written to the network analyzer using this board. The board also allows for control of two coaxial switches located at the antennas. The commands for the switches are sent to a Hewlett Packard switch driver that drives the control signals for both switches. The switches allow for the use of dual-polarized antennas. The second radar system is a Sensors and Software pulseEKKO 1000.

Two sets of antennas will be used. First, two Tecom dual circular sinuous antennas will be used. Their frequencies of operation are from $2 \mathrm{GHz}$ to $18 \mathrm{GHz}$, and the coaxial switches will use both circular polarizations. A pair of loaded dipole antennas will also be used. Their frequencies of operation are from $450 \mathrm{MHz}$ to $1350 \mathrm{MHz}$, and they are provided with the pulseEKKO 1000.

With the above equipment, the entire experiment can be preprogrammed and left alone to execute. The programming for the antenna-positioning system and the radar system is fully integrated using Labview. The result is an operational ground-penetrating radar system that is fully integrated in both hardware and software.

\section{PROCESSING METHODS}

Several post-processing techniques will be evaluated using the ground-penetrating radar test facility. Migration techniques, interferomic SAR, Born-iterative method, and diffraction tomography will all be applied, and the results compared.

The migration techniques include FK migration. In this method the time responses along a single path are collected. The two-dimensional Fourier transform of the time responses and of the position along the path puts the data into the FK domain where $F$ is for frequency and $K$ is for spatial frequency. The FK domain is filtered in order to focus the returns from a single point [1].
The SAR-processing methods include Interferomic SAR. Interferomic SAR applies the phase difference between two baseline traces in order to locate a scatterer in three dimensions, rather than only two as in conventional SAR processing [2].

The Born-iterative technique uses the Born approximation to linearize the scattering equation with respect to the object profile. Once an initial reconstruction of the object profile is obtained, it is used to solve the forward scattering problem. The inverse scattering problem is then solved again using the new information. A new object profile is obtained, and the iterations continue until the object profile converges [3], [4], [5].

Diffraction tomography is another technique that will be investigated. Diffraction tomography relates the twodimensional Fourier transform of the scattered field within a plane to the three-dimensional Fourier transform of the object along a hemispherical arc. The object is illuminated with a plane wave that can be synthesized using stacking or other techniques [5], [6].

\section{CONCLUSIONS}

A new experimental facility for testing subsurface imaging algorithms has been presented and described, as well as the justification for such a site. The facility is fully automated and will allow for ease of data acquisition in a controlled manner. Plans for testing existing algorithms have been presented. Each algorithm will be implemented, and the results compared along with other factors such as computational and data acquisition requirements. Experiments are now underway and results are expected soon. A full investigation of the applicability of ground-penetrating radar imaging techniques will follow.

\section{REFERENCES}

[1] Stolt, R.H., "Migration by Fourier Transform," Geophysics, vol. 43, No. 1, pp. 23-48, 1978.

[2] Allen, C.T., "Interferometric Synthetic Aperture Radar," IEEE Geosci. \& Rem. Sen. Newsletter, issue 96, pp. 6-13, Sept. 1995.

[3] Chew, W.C., Waves and Fields in Inhomogeneous Media, Van Nostrand Reinhold, New York, 1990.

[4] Moghaddam, M., and Chew, W.C., "Nonlinear TwoDimensional Velocity Profile Inversion Using Time Domain Data," IEEE Trans. On Geosci. \& Rem. Sen., vol. 30 , no. 1, pp. 147-156, 1992.

[5] Chaturvedi, P., "Electromagnetic imaging and classification of subsurface targets," Ph. D. Dissertation, University of Kansas, 1995.

[6] Devaney, A.J., "Geophysical Diffraction Tomography," IEEE Trans. On Geosci. \& Rem. Sen., vol. GE-22, no.1, pp. 3-13, January 1984. 